\title{
Coulisses
}

Revue de théâtre

44 | Printemps 2012

Le Hors-scène

\section{L'Échange, de Paul Claudel, seconde version}

Mise en scène de Bernard Lévy, Centre Dramatique National, 12, 13 et 14 avril 2011

\section{Pascal Lécroart}

\section{(2) OpenEdition}

12 Journals

Édition électronique

URL : https://journals.openedition.org/coulisses/487

DOI : $10.4000 /$ coulisses. 487

ISSN : 2546-9460

Éditeur

Presses universitaires de Franche-Comté

\section{Édition imprimée}

Date de publication : 1 juin 2012

Pagination : 173-176

ISBN : 978-2-84867-425-4

ISSN : $1150-594 \mathrm{X}$

\section{Référence électronique}

Pascal Lécroart, "L'Échange, de Paul Claudel, seconde version », Coulisses [En ligne], 44 | Printemps

2012, mis en ligne le 30 novembre 2016, consulté le 29 décembre 2022. URL : http://

journals.openedition.org/coulisses/487 ; DOI : https://doi.org/10.4000/coulisses.487 


\section{L'Échange, seconde version, de Paul Claudel}

Mise en scène de Bernard Lévy, Centre Dramatique National, 12, 13 et 14 avril 2011

PASCAL LÉCROART

Université de Franche-Comté

En 1893, à vingt-cinq ans, aux États-Unis où il est vice-consul, Paul Claudel commence la rédaction de L'Échange qu'il achève un an plus tard. Après Tête d'Or et La Ville, deux drames à la tonalité épique, le jeune auteur s'illustre dans une dramaturgie resserrée : deux couples qui se confrontent et s'échangent en l'espace de 24 heures dans un même lieu. Montée en 1914 par Jacques Copeau, reprise régulièrement par Georges Pitoëff jusqu'aux années quarante, la pièce s'est progressivement imposée aujourd'hui comme le drame le plus joué de Claudel. Les metteurs en scène ont de plus le choix entre deux versions : en 1951, alors que Jean-Louis Barrault se propose de monter la pièce, Claudel ne résiste pas à l'envie de reprendre son texte, presque soixante ans après son achèvement. Les grandes lignes de l'intrigue n'évoluent pas : Louis Laine, jeune homme rimbaldien de sang indien, a épousé lors d'un voyage en France Marthe, d'origine paysanne, à peine plus âgée que lui. La pièce commence lorsque Louis, ayant ramené Marthe aux États-Unis avec lui, a trouvé un emploi de gardien dans la propriété de Thomas Pollock Nageoire, riche financier, marié à une actrice, Lechy Elbernon. Or, Louis a couché la nuit dernière avec Lechy, tandis que Thomas Pollock Nageoire, séduit par la jeune fille, propose à Louis Laine de partir contre une forte somme d'argent. À l'acte II, Louis Laine quitte Marthe au profit de Lechy qui aura surtout été pour lui le prétexte à se libérer des liens du mariage dont il ne veut plus. À l'acte III, victime de la jalousie possessive de Lechy, Louis trouve la mort en voulant s'enfuir, tandis que Marthe fait un geste de réconciliation avec Thomas Pollock Nageoire, geste qu'on peut interpréter comme une promesse d'alliance, même si le texte reste volontairement ambigu. Soucieux de renforcer la valeur dramatique de son texte, Claudel, en 1951, a développé les indications de jeu de scène, réécrit le dialogue dans un style parfois familier, coupé dans la "végétation poétique » et, 
surtout, enrichi le texte d'autres données: Louis Laine ne prend plus l'argent offert par Thomas Pollock Nageoire : il reste sous une pierre ; surtout, Marthe est enceinte et, contrairement à la première version, semble accepter le départ de Louis dont l'enfant vit en elle ; le personnage, que Claudel craignait geignard à la scène, gagne en force et assurance. La pièce y trouve une plus grande valeur dramatique mais perd l'épure poétique de la première version qui est, aujourd'hui, plus fréquemment montée. Il n'est pas forcément évident d'accepter les brutales ruptures de ton de la seconde version.

Pour sa mise en scène, c'est pourtant cette seconde version qu'a choisie Bernard Lévy, jouant des potentialités plus modernes que lui offre le texte. Comme dans la mise en scène de Philippe Sireuil en 1990 au Théâtre Varia de Bruxelles, il choisit d'inscrire très clairement la pièce dans l'Amérique de la fin des années cinquante : le logement du jeune couple est constitué par une petite caravane métallique, légèrement décentrée à gauche, propice à différents jeux de scène, avec une table de camping qui lui est accolée et quelques chaises. Le rimbaldien Louis Laine, au sang indien, devient ainsi une sorte de beatnik en proie au désir d'errance et de découverte. Le reste du plateau est nu, couvert de terre, fermé en arrière plan par une toile plastique transparente, à peine réfléchissante, suggérant la mer. Côté cour, en hauteur, incliné, un vaste écran servant de décor mobile : y sont projetées différentes images du ciel permettant de suivre le déroulement du temps. Une balançoire, idée suggérée par le hamac de la mise en scène de Copeau et que Claudel avait intégrée dans sa seconde version, descendra deux fois brièvement des cintres en cours de $2^{\mathrm{e}}$ et $3^{\mathrm{e}}$ actes.

Bernard Lévy et Jean-Luc Vincent, son assistant à la mise en scène, expliquent, dans le programme, avoir recherché « l'articulation la plus juste entre abstraction symbolique, théâtralité affichée et naturalisme cinématographique ». Cette seconde version, qui laisse subsister des bribes de la première version mettant à mal parfois la cohérence de l'ensemble - le jeune Louis Laine alterne une expression familière avec un emploi parfaitement maîtrisé du subjonctif plus-que-parfait! - se prête très bien à cette lecture. Marthe et Louis Laine apparaissent comme un jeune couple d'un monde réaliste où on s'embrasse, se tient dans les bras, écoute la radio, boit ou fume. On pense, comme le suggère le programme, à Tennessee Williams, en particulier dans l'acte II où Lechy, avec une cruauté méchante, vient provoquer Marthe et oblige Louis Laine à avouer publiquement son choix. Mais ce réalisme coexiste avec des moments de mise à distance : l'écran sert parfois à projeter des bribes du texte et propose une photographie d'un gratte-ciel lorsque Thomas Pollock Nageoire vient présenter son métier. Lechy Elbernon, l'actrice, prend régulièrement les spectateurs à témoin, en particulier dans la célèbre définition du théâtre qu'elle propose à l'acte I. Y a-t-il encore de la place pour le symbolisme ? La mise en scène n'appuie jamais cette perspective. L'écran suggère l'évolution temporelle, mais la scène, même à l'heure de midi, n'est jamais entièrement lumineuse et 
reste le plus souvent dans une demi-pénombre. Il faut chercher essentiellement du côté de certains gestes ou attitudes. Le metteur en scène, sur ce point, n'a pas forcément suivi les nombreuses didascalies de la version de 1951 : si Louis Laine est nu au début, il ne le reste pas jusqu'à l'arrivée de Lechy et Thomas, ni ne joue d'une serviette pour se cacher, comme le mentionne le texte; même si Thomas porte un chapeau, ce n'est pas l'artificiel haut-de-forme indiqué, et Lechy ne le fera pas tomber à la fin du premier acte. On retient en revanche certains gestes forts : Marthe portée dans les bras de Louis et se blottissant contre lui à l'acte $\mathrm{I}$; à l'inverse, Mathe saisissant Louis par derrière pour l'entourer de ses bras à l'acte II, ou le jeu à deux sur la balançoire dans le même acte. On garde aussi en mémoire l'attitude finale très belle : ayant saisi le cadavre de Louis dans ses bras, Marthe maintient sa posture lors de la tirade de Lechy, même lorsqu'elle tend la main à Thomas Pollock Nageoire. La formule de la comptine qui clôt le premier acte, «Akkeri ekkeri ukeri an!» revient alors pour conclure la pièce et marque l'acceptation d'un hasard qui se confond ici avec la Providence. Tout cela témoigne d'un travail attentif et intelligent sur le texte, ce qui est confirmé par l'attention portée au verset. Ses enjambements peuvent aussi bien entrer dans des jeux de scène réalistes, comme, au début de la pièce, lorsque Marthe et Louis prennent leur café du matin, ou servir la diction expressionniste du texte, lors des fameuses considérations de Thomas sur son métier et sur l'économie. Le verset perd en force poétique ce qu'il gagne en valeur dramatique.

On est globalement séduit par le choix et l'interprétation des acteurs. Audrey Bonnet, au corps frêle et aux traits du visage abrupts et peu gracieux, exprime à la fois la fragilité du personnage et sa ténacité farouche qui la fait finalement et paradoxalement victorieuse; manque sans doute en revanche le rayonnement intérieur d'un personnage au pouvoir attractif : c'est ainsi que les trois autres personnages viennent comme se confesser à elle dans le dernier acte. Pierric Plathier, dans le rôle de Louis Laine, privé physiquement de la sauvagerie qu'on attend du personnage, joue sobrement son rôle de victime qui sait que la mort l'attend. Le couple Thomas Pollock Nageoire - Lechy Elbernon, interprété par Pierre-Alain Chapuis et Aline le Berre, est très réussi. La sensualité de la vamp Lechy est bien rendue, y compris dans le délicat passage de la fin de l'acte II où elle déclame la légende de l'enfant au sourcil de pierres, accompagnée par Louis Laine à la guitare. L'évolution du personnage est habilement signalée par son costume : sa tenue élégante des deux premiers actes laisse place au costume du papillon nocturne Acherontia Antropos au début de l'acte III, avant qu'elle n'apparaisse finalement avec la même tunique fine et bon marché que Marthe pour conclure. Lechy, qui revendique le pouvoir de jouer le rôle de Marthe mieux qu'elle, semble ainsi subir in fine la même épreuve du dépouillement, tandis que le costume de Marthe s'est, lui, en revanche enrichi d'un jean. 
Les tenants du théâtre de Claudel peuvent être désarçonnés par cette version tardive du poète à laquelle Pascale Alexandre-Bergues a rendu justice dans sa récente édition critique ${ }^{1}$. Il est intéressant de voir comment elle est pourtant susceptible d'intéresser des metteurs en scène actuels, capables de la porter jusqu'au bout, intelligemment et sobrement, en lui conférant une forme d'actualité frappante. Ce texte d'un dramaturge de plus de quatre-vingts ans étonne en effet par sa verdeur: la nudité initiale de Louis fait toujours son effet, et le texte joue d'allusions sexuelles rendues ici très explicites. L'interrogation que porte le texte sur l'amour, sur la fidélité, sur le mariage, sur le divorce, mais aussi plus généralement sur les relations entre les hommes, qu'elles soient sentimentales, sociales ou économiques, conserve toute son actualité et passe assurément plus facilement auprès d'un public peu averti sur Claudel que le texte de 1893-94. En jouant totalement la carte de cette version, y compris dans ses passages comme la lettre au curé, bien loin du somptueux monologue de la première version, mais parfaitement pensé et intégré dans le réalisme de cette réalisation, le metteur en scène a su rendre justice à cet autre visage de l'œuvre claudélienne qu'on aurait tort de mépriser ou d'ignorer.

1. Édition publiée par les Annales littéraires de l'Université de Franche-Comté, 2002. 